\title{
MONOTONE UTILITY CONVERGENCE
}

\author{
STEFAN ANKIRCHNER, ${ }^{*}$ Imperial College London
}

\begin{abstract}
We show that the maximal expected utility satisfies a monotone continuity property with respect to increasing information. Let $\left(g_{t}^{n}\right)$ be a sequence of increasing filtrations converging to $(g+\infty)$, and let $u^{n}(x)$ and $u^{\infty}(x)$ be the maximal expected utilities when investing in a financial market according to strategies adapted to $\left(g_{t}^{n}\right)$ and $\left(g_{t}^{\infty}\right)$, respectively. We give sufficient conditions for the convergence $u^{n}(x) \rightarrow u^{\infty}(x)$ as $n \rightarrow \infty$. We provide examples in which convergence does not hold. Then we consider the respective utility-based prices, $\pi_{n}$ and $\pi_{\infty}$, of contingent claims under $\left(g_{t}^{n}\right)$ and $\left(g_{t}^{\infty}\right)$. We analyse to what extent $\pi_{n} \rightarrow \pi_{\infty}$ as $n \rightarrow \infty$.

Keywords: Utility maximisation; information; enlargement of filtrations; utility-based price; indifference price
\end{abstract}

2000 Mathematics Subject Classification: Primary 91B16

Secondary $60 \mathrm{H} 30$

\section{Introduction}

The decisions of an investor in a financial market strongly depend on the information he has access to. Naturally, the questions arise of how the behaviour changes if the investor obtains additional information and how strong on average this change will be. Intuitively, we expect that if the (amount of) information increases only slightly, then the optimal investment will not change much either. Put differently, if the information converges then the optimal investment and the related maximal expected utility will converge also. In this paper we first aim at providing sufficient conditions for this convergence. We can interpret the monotone convergence property as information robustness of the financial market model we consider. In a second step, we look at the information dependence of utility-based prices of contingent claims. We will analyse to what extent the prices satisfy a continuity property under increasing information.

We will model the information dependence of optimal investment by using different filtrations to which the investment strategies have to be adapted. This technique has been widely used to model insiders on financial markets (see, e.g. [8] and [12]). Summaries of main results concerning enlargements of filtrations can be found in [10] and [11].

Here is a rough outline of the results. Let $\left(g_{t}^{n}\right)$ be a sequence of increasing filtrations and denote by $\left(g_{t}^{\infty}\right)$ their union. Let $u^{n}(x)$ and $u^{\infty}(x)$ be the suprema of the expected utility when investing in a continuous financial market according to standard sets of strategies adapted to $\left(g_{t}^{n}\right)$ and $\left(g_{t}^{\infty}\right)$, respectively. We will show, under some weak assumptions depending on the type of the utility function, that $u^{n}(x)$ converges to $u^{\infty}(x)$ as $n \rightarrow \infty$.

Let $u^{n}(x, B)$ and $u^{\infty}(x, B)$ be the suprema of the expected utility if, in addition, there is a contingent claim $B$ in the portfolio. Again under some natural conditions, $u^{n}(x, B)$ converges to $u^{\infty}(x, B)$ as $n \rightarrow \infty$. We provide examples in which convergence does not hold.

Received 28 March 2006; revision received 15 June 2006.

* Postal address: Department of Mathematics, Imperial College London, London SW7 2AZ, UK.

Email address: s.ankirchner@imperial.ac.uk 
Finally, we consider the respective utility-based prices, $\pi_{n}$ and $\pi_{\infty}$, of contingent claims under $\left(g_{t}^{n}\right)$ and $\left(g_{t}^{\infty}\right)$. We analyse to what extent $\pi_{n} \rightarrow \pi_{\infty}$ as $n \rightarrow \infty$.

\section{Preliminaries}

Let $(\Omega, \mathcal{F}, \mathrm{P})$ be a probability space and let $S: \Omega \times[0, T] \rightarrow \mathbb{R}$ be a continuous stochastic process starting at 0 . We interpret $S$ as a price process and $T$ as the time horizon. Suppose that $\left(\mathcal{F}_{t}\right)$ is a filtration satisfying the usual conditions. If $S$ is a semimartingale with respect to $\left(\mathcal{F}_{t}\right)$, we denote by $\mathcal{A}(\mathcal{F})$ the set of all $\left(\mathcal{F}_{t}\right)$-predictable processes $\theta$ which satisfy $\theta_{0}=0$ and which are integrable with respect to $S$ and $\left(\mathcal{F}_{t}\right)$ in the usual sense (see, e.g. [13, p. 165]). The elements of $\mathcal{A}(\mathcal{F})$ will be called strategies. Moreover, a strategy is called $a$-admissible if the stochastic integral process, denoted by $(\theta \cdot S)$, satisfies $(\theta \cdot S)_{t} \geq-a$ for all $t \in[0, T]$. More generally, $\theta$ will be called admissible if it is $a$-admissible for some $a$. Finally, we say that $S$ satisfies the no-arbitrage $(N A)$ condition with respect to $\left(\mathcal{F}_{t}\right)$ if there exists no admissible $\theta \in \mathcal{A}(\mathcal{F})$ such that $(\theta \cdot S)_{T} \geq 0$ and $\mathrm{P}\left((\theta \cdot S)_{T}>0\right)>0$.

By a utility function $U$ we mean any concave function $U: \mathbb{R} \rightarrow \mathbb{R} \cup\{-\infty\}$. The maximal expected utility with respect to $\left(\mathcal{F}_{t}\right)$ is defined by

$$
u^{\mathcal{F}}(x)=\sup \left\{\mathrm{E} U\left(x+(\theta \cdot S)_{T}\right): \theta \in \mathcal{A}(\mathcal{F}) \text { is admissible }\right\} .
$$

We will also consider the function

$$
u_{a}^{\mathcal{F}}(x)=\sup \left\{\mathrm{E} U\left(x+(\theta \cdot S)_{T}\right): \theta \in \mathcal{A}(\mathcal{F}) \text { is } a \text {-admissible }\right\} .
$$

Now let $\left(g_{t}^{n}\right)$ be a sequence of increasing filtrations satisfying the usual conditions. Moreover, suppose that $S$ is a semimartingale relative to any $\left(g_{t}^{n}\right)$. The smallest filtration satisfying the usual conditions and containing every filtration $\left(g_{t}^{n}\right)$ will be denoted by

$$
g_{t}^{\infty}=\bigcap_{s>t} \bigvee_{n \geq 1} g_{s}^{n}
$$

Throughout, we suppose that $S$ is a continuous $\left(g_{t}^{\infty}\right)$-semimartingale with decomposition

$$
S_{t}=M_{t}+\int_{0}^{t} \alpha_{s} \mathrm{~d}\langle M, M\rangle_{s},
$$

where $M$ is a $\left(g_{t}^{\infty}\right)$-local martingale starting at $0,\langle M, M\rangle$ is the quadratic variation of $M$, and $\alpha$ is a $\left(g_{t}^{\infty}\right)$-predictable process satisfying $\int_{0}^{T} \alpha_{t}^{2} \mathrm{~d}\langle M, M\rangle_{t}<\infty$ almost surely (a.s.). Note that some no-arbitrage-type conditions, e.g. the 'no free lunch with vanishing risk' condition, imply the existence of such a semimartingale decomposition (see [5], [6], and Chapter 9 of [7]). Alternatively, if $\lim _{x \rightarrow \infty} U(x)=\infty$ and $u_{a}^{g \infty}(x)$ is finite for some $a>0$, then there exists a decomposition of the form (2.1) (see [2] and [3] ).

Note that, with respect to every subfiltration $\left(g_{t}^{n}\right)$, we can also find a decomposition of $S=M^{n}+\alpha_{s}^{n} \cdot\langle M, M\rangle$ such that $\alpha^{n}$ is locally square integrable. This guarantees that, for every $\left(g_{t}^{n}\right)$-measurable strategy $\theta$, the stochastic integrals $\left(\theta \cdot g^{n} S\right)$ and $(\theta \cdot g \infty S)$, defined with respect to $\left(g_{t}^{n}\right)$ and $\left(g_{t}^{\infty}\right)$, respectively, are the same. We therefore omit the filtrations in the definition of the integrals.

We denote by $u^{n}(x)$ the maximal expected utility with respect to $\left(g_{t}^{n}\right)$, and by $u^{\infty}(x)$ the maximal expected utility with respect to $\left(g_{t}^{\infty}\right)$. We use the similarly abbreviated notation $u_{a}^{n}(x)$ and $u_{a}^{\infty}(x)$, for $a>0$. 
Since the sequence $\left(g_{t}^{n}\right)$ is increasing, $u^{n}(x)$ is also increasing. We will provide sufficient conditions for the convergence $u^{n}(x) \rightarrow u^{\infty}(x)$ as $n \rightarrow \infty$. We will have to distinguish between two types of our utility functions, on the basis of the so-called domain of $U$, which is defined by $\operatorname{dom}(U)=\{y: U(y)>-\infty\}$. We will first consider the case $\operatorname{dom}(U)=\mathbb{R}$ and then the case $\operatorname{dom}(U) \neq \mathbb{R}$.

\section{Monotone utility convergence}

\subsection{Convergence in the $\operatorname{case} \operatorname{dom}(U)=\mathbb{R}$}

Throughout this subsection we assume that $\operatorname{dom}(U)=\mathbb{R}$. We start with the observation that the utility maximum can be attained by using strategies in

$$
L^{2}(M)=\left\{\theta \text { measurable: } \mathrm{E} \int_{0}^{T} \theta_{t}^{2} \mathrm{~d}\langle M, M\rangle_{t}<\infty\right\} .
$$

For any filtration $\left(\mathcal{F}_{t}\right)$, we denote by $L_{\mathscr{F}}^{2}(M)$ the set of all $\left(\mathcal{F}_{t}\right)$-predictable processes $\theta \in$ $L^{2}(M)$.

Lemma 3.1. Let $\left(\mathcal{F}_{t}\right)$ be a filtration with respect to which $S$ is a semimartingale. Let $x \in \mathbb{R}$ and $a \in(0, \infty)$. Then

$$
u_{a}^{\mathcal{F}}(x)=\sup \left\{\mathrm{E} U\left(x+(\theta \cdot S)_{T}\right): \theta \in L_{\mathcal{F}}^{2}(M) \cap \mathcal{A} \text { is }(a-\varepsilon) \text {-admissible for some } \varepsilon>0\right\} .
$$

Proof. We first prove that, for all $x \in \mathbb{R}$,

$$
u^{\infty}(x)=\sup _{\varepsilon>0} \sup \left\{\mathrm{E} U\left(x+(\theta \cdot S)_{T}\right): \theta \in \mathcal{A}\left(g^{\infty}\right) \text { is }(x-\varepsilon) \text {-admissible }\right\} .
$$

We have to show only that the left-hand side does not exceed the right-hand side. For this, let $\theta \in \mathcal{A}\left(g^{\infty}\right)$ such that $\mathrm{E} U\left(x+(\theta \cdot S)_{T}\right)>-\infty$. Set $\theta^{n}=(1-1 / n) \theta$ for all $n \geq 1$. Clearly, $\theta^{n}$ is $(x-x / n)$-admissible. Monotone convergence applied to the negative and positive parts of $U\left(x+\left(\theta^{n} \cdot S\right)_{T}\right)-U(x)$ implies that

$$
\lim _{n} \mathrm{E} U\left(x+\left(\theta^{n} \cdot S\right)_{T}\right)=\mathrm{E} U\left(x+(\theta \cdot S)_{T}\right)
$$

hence, $u^{\infty}(x)$ is smaller than the right-hand side of (3.2).

Obviously, the right-hand side of (3.1) is not greater than the left-hand side. For the reverse inequality, choose an $\varepsilon>0$ and an $(a-\varepsilon)$-admissible strategy $\theta$ satisfying $\mathrm{E} U\left(x+(\theta \cdot S)_{T}\right)>$ $-\infty$. By (3.2), it is sufficient to show that $\mathrm{E} U\left(x+(\theta \cdot S)_{T}\right)$ is not greater than the right-hand side of (3.1). Since $\theta$ is $S$-integrable, the stopping times

$$
T_{n}=T \wedge \inf \left\{t \geq 0: \int_{0}^{t} \theta_{r}^{2} \mathrm{~d}\langle M, M\rangle_{r} \leq n\right\}
$$

converge almost surely to $T$ for $n \rightarrow \infty$. Note that the strategies

$$
\theta^{n}=\mathbf{1}_{\left[0, T_{n}\right]} \theta
$$

are $(a-\varepsilon)$-admissible and belong to $L_{\mathcal{F}}^{2}(M)$. Fatou's lemma implies that

$$
\liminf _{n} \mathrm{E} U\left(x+\left(\theta^{n} \cdot S\right)_{T}\right) \geq \mathrm{E} U\left(x+(\theta \cdot S)_{T}\right),
$$

from which the result follows. 
Let us return to the filtrations $\left(g_{t}^{n}\right)$ and $\left(g^{\infty}\right)$ introduced in Section 2 . We start by showing that the sequence $\left(u_{a}^{n}(x)\right)$ satisfies a monotone convergence property.

Theorem 3.1. Let $x \in \mathbb{R}$ and $a \in(0, \infty)$. Then $\lim _{n} u_{a}^{n}(x)=u_{a}^{\infty}(x)$.

Proof. Let $\theta \in L_{g \infty}^{2}(M)$ be $(a-\varepsilon)$-admissible. The stopping times

$$
\tau_{k}=T \wedge \inf \left\{t \geq 0: \int_{0}^{t} \alpha_{s}^{2} \mathrm{~d}\langle M, M\rangle_{s} \geq k\right\}
$$

converge to $T$ a.s., and, hence,

$$
\liminf _{k} \mathrm{E} U\left(x+(\theta \cdot S)_{\tau_{k}}\right) \geq \mathrm{E} U\left(x+(\theta \cdot S)_{T}\right) .
$$

By Lemma 3.1, it suffices to show that, for all $k \geq 1$, E $U\left(x+(\theta \cdot S)_{\tau_{k}}\right)$ is not greater than $\sup _{n} u_{a}^{n}(x)$. To simplify our notation we assume that $\tau_{k}=T$ for some $k$.

Now let $\theta^{n}$ be the projection of $\theta$ onto $L_{g^{n}}^{2}$. Note that

$$
\mathrm{E}\left[\left(\left(\theta^{n}-\theta\right) \cdot S\right)_{T}^{*}\right] \leq \mathrm{E}\left[\left(\left(\theta^{n}-\theta\right) \cdot M\right)_{T}^{*}\right]+\mathrm{E}\left[\left(\left(\theta^{n}-\theta\right) \alpha \cdot\langle M, M\rangle\right)_{T}^{*}\right],
$$

where '*' stands for the bilateral supremum (i.e. $X_{t}^{*}=\sup \left\{\left|X_{s}\right|: 0 \leq s \leq t\right\}$ for any process $X$ ). By Doob's $L^{2}$-inequality (see, e.g. Theorem 1.7, Chapter II, of [14]), there is a constant $C>0$ such that

$$
\begin{aligned}
\mathrm{E}\left[\left(\left(\theta^{n}-\theta\right) \cdot S\right)_{T}^{*}\right] & \leq\left(\mathrm{E}\left[\left(\left(\theta^{n}-\theta\right) \cdot M\right)_{T}^{*}\right]^{2}\right)^{1 / 2}+\mathrm{E}\left[\left(\left(\theta^{n}-\theta\right) \alpha \cdot\langle M, M\rangle\right)_{T}^{*}\right] \\
& \leq C\left(\mathrm{E}\left[\left(\left(\theta^{n}-\theta\right) \cdot M\right)_{T}\right]^{2}\right)^{1 / 2}+\mathrm{E}\left[\left(\left|\theta^{n}-\theta\right||\alpha| \cdot\langle M, M\rangle\right)_{T}\right]
\end{aligned}
$$

The first summand in the preceding line tends to 0 as $n \rightarrow \infty$, because $\left(\theta^{n}\right)$ converges to $\theta$ in $L^{2}(M)$. The second vanishes due to the Kunita-Watanabe inequality (see Corollary 1.16, Chaper IV, of [14]) and due to our assumption that $\int_{0}^{T} \alpha_{s}^{2} \mathrm{~d}\langle M, M\rangle_{s}$ is bounded. Consequently, by choosing a subsequence, if necessary, the sequence $\left(\theta^{n} \cdot S\right)$ almost everywhere converges uniformly to $(\theta \cdot S)$ on $[0, T]$. Now set $T_{n}=T \wedge \inf \left\{t \geq 0:\left(\theta^{n} \cdot S\right)_{t} \leq-a\right\}$ and $\pi^{n}=\mathbf{1}_{\left[0, T_{n}\right]} \theta^{n}$. The strategies $\pi^{n}$ are $a$-admissible and satisfy

$$
\lim _{n}\left(\pi^{n} \cdot S\right)_{T}=(\theta \cdot S)_{T} \quad \text { a.s. }
$$

Using Fatou's lemma, we obtain

$$
\liminf _{n} \mathrm{E} U\left(x+\left(\pi^{n} \cdot S\right)_{T}\right) \geq \mathrm{E} U\left(x+(\theta \cdot S)_{T}\right)
$$

and, hence, the result.

We immediately obtain the following corollary.

Corollary 3.1. For all $x \in \mathbb{R}$, we have $\lim _{n} u^{n}(x)=u^{\infty}(x)$.

\subsection{Convergence in the $\operatorname{case} \operatorname{dom}(U) \neq \mathbb{R}$}

Throughout this subsection we assume that $\operatorname{dom}(U) \neq \mathbb{R}$. To simplify our notation we suppose that $\sup \{y: U(y)=-\infty\}=0$. The analogue of Lemma 3.1 is as follows. 
Lemma 3.2. For $x>0$ and $a \in(0, x]$, we have

$$
u_{a}^{\mathcal{F}}(x)=\sup \left\{\mathrm{E} U\left(x+(\theta \cdot S)_{T}\right): \theta \in L_{\mathcal{F}}^{2}(M) \text { is }(a-\varepsilon) \text {-admissible for some } \varepsilon>0\right\} .
$$

Proof. This can be proved in the way Lemma 3.1 was. Notice that Fatou's lemma can only be applied if $a \leq x$.

From this we can deduce the analogue of Theorem 3.1.

Theorem 3.2. For $x>0$ and $a \in(0, x]$, we have $\lim _{n} u_{a}^{n}(x)=u_{a}^{\infty}(x)$.

Proof. The proof is similar to that of Theorem 3.1. We have only to replace the stopping times $T_{n}$ by $T_{n}^{\prime}=T \wedge \inf \left\{t \geq 0:\left(\theta^{n} \cdot S\right)_{t} \leq-a+\varepsilon / 2\right\}$. Then the strategies $\pi^{n}=\mathbf{1}_{\left[0, T_{n}^{\prime}\right]} \theta^{n}$ are $(a-\varepsilon / 2)$-admissible, and we again apply Fatou's lemma.

We will see in Example 3.1 that Theorem 3.2 is not valid without the assumption that $a \in(0, x]$. However, we can do without this assumption if $S$ satisfies the NA condition. To prove this we need the following result.

Lemma 3.3. Let $a>0$ and $\theta \in \mathcal{A}\left(g^{\infty}\right)$. Suppose that $S$ satisfies the NA condition relative to $\left(g_{t}^{\infty}\right)$. If $(\theta \cdot S)_{T} \geq-a$ a.s., then $\theta$ is a-admissible.

Proof. For every $\varepsilon>0$, define a stopping time by

$$
\tau_{\varepsilon}=\inf \left\{t>0:(\theta \cdot S)_{t}=-a-\varepsilon\right\} \wedge T \text {. }
$$

Suppose that $\theta$ is not $a$-admissible. Then, for some $\varepsilon>0$, we must have $\mathrm{P}\left(\tau_{\varepsilon}<T\right)>$ 0 . The strategy $\pi=\mathbf{1}_{\left(\tau_{\varepsilon}, T\right]} \theta$ satisfies $(\pi \cdot S)_{T}=\mathbf{1}_{\left\{\tau_{\varepsilon}<T\right\}}\left[(\theta \cdot S)_{T}-(\theta \cdot S)_{\tau_{\varepsilon}}\right] \geq 0$, and $\mathrm{P}\left((\pi \cdot S)_{T}>0\right)=\mathrm{P}\left(\tau_{\varepsilon}<T\right)>0$. Hence, $\pi$ is an arbitrage opportunity, in contradiction to the NA condition.

Corollary 3.2. If $S$ satisfies the NA condition relative to $\left(g_{t}^{\infty}\right)$, then, for all $x>0$, we have $\lim _{n} u^{n}(x)=u^{\infty}(x)$.

Proof. Let $\theta$ be a $\left(g_{t}^{\infty}\right)$-predictable strategy such that $\mathrm{E} U\left(x+(\theta \cdot S)_{T}\right)>-\infty$. Then $(\theta \cdot S)_{T} \geq-x$ a.s., Lemma 3.3 implies that $\theta$ is $x$-admissible, and we thus have $u^{\infty}(x)=$ $u_{x}^{\infty}(x)$. Similarly, we obtain $u^{n}(x)=u_{x}^{n}(x), n \geq 1$. The claim now follows from Theorem 3.2.

The next example shows that imposition of the NA condition is necessary in Corollary 3.2.

Example 3.1. Let $W$ be a Brownian motion with respect to $\left(\mathcal{F}_{t}\right)$ and suppose that the price process is given by $S_{t}=S_{0}+W_{t}$, with $S_{0}>0$ a constant. Moreover, let $\left(\psi_{n}\right)$ be an independent, identically distributed sequence of random variables with standard normal distribution $\mathrm{N}(0,1)$, independent of $\left(\mathcal{F}_{t}\right)$. Let $G=\mathbf{1}_{(1,2)}\left(W_{T}\right)$ and $G_{n}=G+\psi_{n}$. We consider the increasing sequence of filtrations

$$
g_{t}^{n}=\bigcap_{s>t} \sigma\left(G_{1}, \ldots, G_{n}\right) \vee \mathcal{F}_{s}
$$

and set $g_{t}^{\infty}=\bigcap_{s>t} \bigvee_{n} g_{s}^{n}$. Notice that $g_{0}^{\infty}$ contains all the information in $G$, since, owing to the law of large numbers, $\lim _{n}(1 / n)\left(G_{1}+\cdots+G_{n}\right)=G$ a.s. As a consequence, under $\left(g_{t}\right)$ there is arbitrage and, hence, $u^{\infty}(x)=\infty$. 
Under $\left(g_{t}^{n}\right)$, however, there are no arbitrage opportunities, since it is possible to construct an equivalent local martingale measure for $\left(g_{t}^{n}\right)$. Let $\pi_{t}\left(\left(G_{1}, \ldots, G_{n}\right) \in \mathrm{d} a, \omega\right)$ be a regular conditional distribution with respect to $\mathcal{F}_{t}$. Then, for almost all $\omega$, we have

$$
\pi_{t}\left(\left(G_{1}, \ldots, G_{n}\right) \in \mathrm{d} a, \omega\right) \sim \mathrm{P}_{\left(G_{1}, \ldots, G_{n}\right)}(\mathrm{d} a),
$$

where $\mathrm{P}_{\left(G_{1}, \ldots, G_{n}\right)}$ is the joint distribution of $\left(G_{1}, \ldots, G_{n}\right)$. It follows from known results (see, e.g. [1] and [9]) that there exists an equivalent measure under which the vector $\left(G_{1}, \ldots, G_{n}\right)$ is independent of $\mathcal{F}_{T}$; hence, there exists an equivalent local martingale measure for $S$ under the filtration $\left(g_{t}^{n}\right)$.

The preceding argument shows that the NA condition holds under $\left(g_{t}^{n}\right)$. Therefore, $u^{n}(x)=$ $u_{x}^{n}(x), n \geq 1$. Now, $u_{x}^{n}(x) \leq u_{x}^{\infty}(x)$. For $U(x)=\log (x)$, it has been shown in [4] that $u_{x}^{\infty}(x)$ is equal to the mutual information between $G$ and $S$, which is given by $I(G, S)=$ $p \log p+(1-p) \log (1-p)$, where $p=\mathrm{P}(G=1)$. Consequently, the sequence $u^{n}(x)$ is bounded by $I(G, S)<\infty$, which shows that $\lim _{n} u^{n}(x) \neq u^{\infty}(x)$.

Finally, observe that $\sup _{a>0} u_{a}^{\infty}(x)=u^{\infty}(x)=\infty$. Therefore, there exists an $a>0$ and an $a$-admissible strategy $\theta$ such that $\mathrm{E} U\left(x+(\theta \cdot S)_{T}\right)>I(G, S)$. Consequently, $u_{a}^{\infty}(x)>$ $I(G, S) \geq \lim \sup _{n} u_{a}^{n}(x)$, which shows that Theorem 3.2 does not hold without the assumption that $a \in(0, x]$.

\subsection{Why not use arbitrary price processes?}

We close this section with an example showing that Theorems 3.1 and 3.2 and Corollaries 3.1 and 3.2 are not valid without some regularity assumption being made about $S$.

Example 3.2. Let $T>1$ and let $\phi$ be a random variable with standard normal distribution $\mathrm{N}(0,1)$. Suppose that the price process is given by

$$
S_{t}= \begin{cases}1 & \text { if } 0 \leq t<1, \\ 1+\phi+\frac{1}{2} & \text { if } 1 \leq t \leq T,\end{cases}
$$

and let $\left(\mathcal{F}_{t}^{S}\right)$ be the completed filtration generated by $S$. Moreover, let $\left(\varepsilon_{n}\right)$ be a sequence of independent normal random variables with mean 0 and $\operatorname{var}\left(\varepsilon_{n}\right)=1 / n$. Let $\xi_{n}=\mathbf{1}_{\{|\phi| \geq 1\}}+\varepsilon_{n}$ and $g_{t}^{n}=\mathcal{F}_{t} \vee \sigma\left(\xi_{1}, \ldots, \xi_{n}\right), 0 \leq t \leq T$. We claim that $u^{n}(x)=U(x)$ for all $x>0$. To prove this, let $\theta$ be $\left(g g_{t}^{n}\right)$-predictable and $S$-integrable. If $\theta_{1} \neq 0$ a.s., then the integral $(\theta \cdot S)_{1}$ is unbounded from below and, hence, $\theta$ is not admissible. Since the process $S$ is constant on the remaining part of the trading interval, we have $u_{a}^{n}(x)=U(x)$. A trader having access to the information represented by $g_{t}^{\infty}=\bigvee_{n \geq 1} g_{t}^{n}$ knows whether the absolute value of $\phi$ is bigger or smaller than 1. Therefore, he has access to nontrivial admissible trading strategies. As a consequence, $u^{\infty}(x)>U(x)$ and, hence,

$$
\lim _{n} u_{a}^{n}(x) \neq u_{a}^{\infty}(x), \quad \lim _{n} u^{n}(x) \neq u^{\infty}(x) .
$$

Note that the price process $S$ satisfies the NA condition with respect to $\left(g_{t}^{\infty}\right)$. Therefore, the assumption that $S$ is continuous cannot be dropped in Corollary 3.2.

\section{Monotone convergence with random endowments}

We aim to prove a continuity property of utility-based prices of contingent claims. To this end we have to generalise the results of the previous section to the case where the terminal 
wealth of an investor is not only determined by his investment strategy but also by a random payment or endowment due at time $T$.

Let $B$ be a random variable (endowment). For any semimartingale filtration $\left(\mathscr{H}_{t}\right)$, we define

$$
u^{\mathscr{H}}(x, B)=\sup \left\{\mathrm{E} U\left(x+(\theta \cdot S)_{T}+B\right): \theta \in \mathcal{A}(\mathscr{H})\right\} .
$$

We will analyse to what extent the maximal expected utility with random endowment $B$ has the monotone convergence property. For this, let $\left(g_{t}^{n}\right)$ again be an increasing sequence with limit $\left(g_{t}^{\infty}\right)$, in which case the assumptions of Section 2 are satisfied. As above, we will use the abbreviated notation $u^{n}(x, B) \equiv u^{g^{n}}(x, B)$ and $u^{\infty}(x, B) \equiv u^{g^{\infty}}(x, B)$.

\subsection{Convergence in the $\operatorname{case} \operatorname{dom}(U)=\mathbb{R}$}

We need an integrability condition on our random endowment. We use the following one:

$$
\mathrm{E} U(B+a)>-\infty \text { for all } a \in \mathbb{R} .
$$

Note that if $U$ is the exponential utility function, then the integrability of $U\left(B+a_{0}\right)$ for one point $a_{0} \in \mathbb{R}$ suffices to imply condition (4.1).

Theorem 4.1. If (4.1) holds then $\lim _{n} u^{n}(x, B)=u^{\infty}(x, B)$.

Proof. Let $\theta$ be $\left(g_{t}^{\infty}\right)$-predictable and admissible, say $a$-admissible. Note that (4.1) implies $\mathrm{E} U\left(x+(\theta \cdot S)_{T}+B\right)>-\infty$. We can find $\left(g_{t}^{n}\right)$-predictable strategies $\theta^{n}$ such that $\left(\theta^{n} \cdot S\right) \rightarrow$ $(\theta \cdot S)$ uniformly on $[0, T]$ a.s. By stopping at $\tau_{n}=\inf \left\{t \geq 0:\left|\left(\left(\theta^{n}-\theta\right) \cdot S\right)_{t}\right| \geq \delta\right\}$, where $\delta>0$, we obtain $(a+\delta)$-admissible strategies $\pi^{n}=\mathbf{1}_{\left[0, \tau^{n}\right]} \theta^{n}$ for which $\left(\pi^{n} \cdot S\right)_{T} \rightarrow(\theta \cdot S)_{T}$ a.s. and $x+\left(\pi^{n} \cdot S\right)_{T}+B \geq x-a-\delta+B$. Since the negative part of $U(x-a-\delta+B)$ is integrable, the negative parts of $U\left(x+\left(\pi^{n} \cdot S\right)_{T}+B\right)$ are uniformly integrable. Therefore,

$$
\lim _{n} \mathrm{E}\left[U\left(x+\left(\pi^{n} \cdot S\right)_{T}+B\right)\right]^{-}=\mathrm{E}\left[U\left(x+(\theta \cdot S)_{T}+B\right)\right]^{-} .
$$

Consequently, by Fatou's lemma,

$$
\begin{aligned}
& \liminf _{n} \mathrm{E} U\left(x+\left(\pi^{n} \cdot S\right)_{T}+B\right) \\
& \quad=\liminf _{n} \mathrm{E}\left[U\left(x+\left(\pi^{n} \cdot S\right)_{T}+B\right)\right]^{+}-\lim _{n} \mathrm{E}\left[U\left(x+\left(\pi^{n} \cdot S\right)_{T}+B\right)\right]^{-} \\
& \quad \geq \mathrm{E}\left[U\left(x+(\theta \cdot S)_{T}+B\right)\right]^{+}-\mathrm{E}\left[U\left(x+(\theta \cdot S)_{T}+B\right)\right]^{-} \\
& \quad=\mathrm{E} U\left(x+(\theta \cdot S)_{T}+B\right)
\end{aligned}
$$

and, hence, $\liminf _{n} u^{n}(x, B) \geq u^{\infty}(x, B)$. Since obviously $u^{n}(x, B) \leq u^{\infty}(x, B)$, the result follows.

\subsection{Convergence in the case $\operatorname{dom}(U) \neq \mathbb{R}$}

Throughout this subsection we again assume that $\sup \{y: U(y)=-\infty\}=0$. We start by showing that, in this case, a monotone convergence result like Theorem 4.1 may no longer be true (even if there is no arbitrage under $\left(g_{t}^{\infty}\right)$ ).

Example 4.1. Let $W$ be a Brownian motion with respect to a filtration $\left(\mathscr{F}_{t}\right)$, let $\mathrm{d} S_{t}=S_{t}\left(\mathrm{~d} W_{t}+\right.$ $\alpha \mathrm{d} t)$ with $S_{0}>0$ and $\alpha>0$, let $B$ be independent of $\left(\mathcal{F}_{t}\right)$ and such that $\mathrm{P}(B=-1)=\frac{1}{2}=$ $\mathrm{P}(B=1)$, and let $\left(V_{t}\right)$ be another Brownian motion which is independent of $B$ and $\left(\mathcal{F}_{t}\right)$. Let 
$\mathscr{H}^{n}=\sigma\left(B+V_{r}: r \geq 1 / n\right)$ and $\mathscr{g}_{t}^{n}=\bigcap_{s>t} \mathscr{H}^{n} \vee \mathcal{F}_{s}$, for $n \geq 1$. The union $\mathcal{G}_{t}^{\infty}=\bigcap_{s>t} \bigvee_{n} g_{s}^{n}$ contains $\sigma(B)$. Now suppose that $U(x)=\log (x)$ and that $x>1$. Under $\left(g_{t}^{n}\right)$, we have

$$
u^{n}(x, B)=u_{x-1}^{n}(x, B) \leq u_{x-1}^{\infty}(x, B)=\frac{1}{2} u_{x-1}^{\mathcal{F}}(x-1)+\frac{1}{2} u_{x-1}^{\mathcal{F}}(x+1) .
$$

Under $\left(g_{t}^{\infty}\right)$, we know from the beginning whether $B=-1$ or $B=1$. Therefore,

$$
u^{\infty}(x, B)=\frac{1}{2} u_{x-1}^{\mathcal{F}}(x-1)+\frac{1}{2} u_{x+1}^{\mathcal{F}}(x+1) .
$$

Note that $u_{x-1}^{\mathcal{F}}(x+1) \neq u_{x+1}^{\mathcal{F}}(x+1)$ and, thus, $\lim _{n} u^{n}(x, B) \neq u^{\infty}(x, B)$.

This example shows that we have to make additional assumptions in order to guarantee the convergence of $u^{n}(x, B)$ to $u^{\infty}(x, B)$. We continue with some auxiliary results that hold for any filtration $\left(\mathcal{F}_{t}\right)$ with respect to which $S$ is a semimartingale.

Lemma 4.1. Let $x>0$ and $a>0$, and suppose that $\mathrm{E} U(B+x)>-\infty$. Then

$$
u_{a}^{\mathcal{F}}(x, B)=\sup _{\varepsilon>0} u_{a-\varepsilon}^{\mathcal{F}}(x, B) .
$$

Proof. Let $\theta$ be an $a$-admissible strategy such that $\mathrm{E} U\left(x+(\theta \cdot S)_{T}+B\right)>-\infty$. Set $\theta^{n}=(1-1 / n) \theta$ for all $n \geq 1$. Clearly $\theta^{n}$ is $(a-a / n)$-admissible. Observe that

$$
\left[U\left(x+\left(\theta^{n} \cdot S\right)_{T}+B\right)-U(x+B)\right]^{-} \leq\left[U\left(x+(\theta \cdot S)_{T}+B\right)-U(x+B)\right]^{-}
$$

and, therefore, that $\left[U\left(x+\left(\theta^{n} \cdot S\right)_{T}+B\right)-U(x+B)\right]^{-}$is uniformly integrable. By applying Fatou's lemma to the positive parts, we obtain

$$
\begin{aligned}
& \liminf _{n} \mathrm{E} U\left(x+\left(\theta^{n} \cdot S\right)_{T}+B\right) \\
&= \liminf _{n} \mathrm{E}\left[U\left(x+\left(\theta^{n} \cdot S\right)_{T}+B\right)-U(x+B)\right]+\mathrm{E} U(x+B) \\
&= \mathrm{E} U(x+B)+\liminf _{n} \mathrm{E}\left[U\left(x+\left(\theta^{n} \cdot S\right)_{T}+B\right)-U(x+B)\right]^{+} \\
&-\lim _{n} \mathrm{E}\left[U\left(x+\left(\theta^{n} \cdot S\right)_{T}+B\right)-U(x+B)\right]^{-} \\
& \geq \mathrm{E} U(x+B)+\mathrm{E}\left[U\left(x+(\theta \cdot S)_{T}+B\right)-U(x+B)\right]^{+} \\
&-\mathrm{E}\left[U\left(x+(\theta \cdot S)_{T}+B\right)-U(x+B)\right]^{-} \\
&= \mathrm{E} U\left(x+(\theta \cdot S)_{T}+B\right) .
\end{aligned}
$$

Therefore, $u_{a}^{\mathcal{F}}(x, B) \leq \sup _{\varepsilon>0} u_{a-\varepsilon}^{\mathcal{F}}(x, B)$. Since the right-hand side of this inequality obviously does not exceed the left-hand side, the proof is complete.

Lemma 4.2. Let $a, b>0$. If $\operatorname{ess} \inf B \geq-b$ then, for all $x \geq a+b$,

$$
\begin{aligned}
& u_{a}^{\mathcal{F}}(x, B)=\sup \left\{\mathrm{E} U\left(x+(\theta \cdot S)_{T}+B\right):\right. \\
& \left.\quad \theta \in L_{g}^{2}(M) \cap \mathcal{A}(\mathcal{F}) \text { is }(a-\varepsilon) \text {-admissible for some } \varepsilon>0\right\} .
\end{aligned}
$$

Proof. Let $\theta \in \mathcal{A}(\mathcal{F})$ be $(a-\varepsilon)$-admissible. The stopping times

$$
T_{n}=T \wedge \inf \left\{t \geq 0: \int_{0}^{t} \theta_{r}^{2} \mathrm{~d}\langle M, M\rangle_{r} \leq n\right\}
$$


converge almost surely to $T$. The strategies $\theta^{n}=\mathbf{1}_{\left[0, T_{n}\right]} \theta$ are $(a-\varepsilon)$-admissible and belong to $L_{g}^{2}(M)$. Fatou's lemma implies that

$$
\liminf _{n} \mathrm{E} U\left(x+\left(\theta^{n} \cdot S\right)_{T}+B\right) \geq \mathrm{E} U\left(x+(\theta \cdot S)_{T}+B\right),
$$

from which, by Lemma 4.1, the result follows.

Theorem 4.2. Let $a, b>0$. If $\operatorname{ess} \inf B \geq-b$ then, for all $x \geq a+b$,

$$
\lim _{n} u_{a}^{n}(x, B)=u_{a}^{\infty}(x, B) .
$$

Proof. Let $\theta \in L_{g}^{2}(M) \cap \mathcal{A}(\xi)$ be $(a-\varepsilon)$-admissible. By Lemma 4.2, it is enough to show that $\lim _{n} u_{a}^{n}(x, B) \geq \mathrm{E} U\left(x+(\theta \cdot S)_{T}+B\right)$. As in the proof of Theorem 3.1, we can construct $(a-\varepsilon / 2)$-admissible and $\left(g_{t}^{n}\right)$-predictable strategies $\pi^{n}$ such that $\left(\pi^{n} \cdot S\right)_{T}$ converges to $(\theta \cdot S)_{T}$ a.s. Fatou's lemma then implies that

$$
\liminf _{n} \mathrm{E} U\left(x+\left(\pi^{n} \cdot S\right)_{T}+B\right) \geq \mathrm{E} U\left(x+(\theta \cdot S)_{T}+B\right),
$$

and the result follows.

If $B$ is independent of the filtration $\left(g_{t}^{\infty}\right)$, we can weaken the assumptions of Theorem 4.2.

Theorem 4.3. Let $B$ be a random variable independent of $\left(g_{t}^{\infty}\right)$. Suppose that ess inf $B=-b$ and $x>b$. If the NA condition holds under $\left(g_{t}^{\infty}\right)$, then $\lim _{n} u^{n}(x, B)=u^{\infty}(x, B)$.

Proof. We first show that $u^{\infty}(x, B)=u_{x-b}^{\infty}(x, B)$. Suppose that $\mathrm{P}\left((\theta \cdot S)_{T}<-x+b\right)>0$. Then, independence of $B$ implies that $x+(\theta \cdot S)_{T}+B<0$ with positive probability and, hence, that $\mathrm{E} U\left(x+(\theta \cdot S)_{T}+B\right)=-\infty$. Therefore, $u^{\infty}(x, B)$ is attained by strategies satisfying $(\theta \cdot S)_{T} \geq-x+b$ almost everywhere. Since the NA condition holds, $\theta$ must be $(x-b)$-admissible. As a consequence, we have $u^{\infty}(x, B)=u_{x-b}^{\infty}(x, B)$. From Lemma 4.1, we obtain $u^{\infty}(x, B)=\sup _{\varepsilon>0} u_{x-b-\varepsilon}^{\infty}(x, B)$. Now we proceed as in the proof of Theorem 4.2.

Note that Example 4.1 shows that the independence assumption cannot be omitted in Theorem 4.3.

\section{Convergence of indifference prices}

Definition 5.1. Let $B$ be a random endowment and suppose that there exists a unique real number $\pi$ such that $u^{\mathscr{H}}(x-\pi, B)=u^{\mathscr{H}}(x)$. Then $\pi$ is called the indifference price or utility-based price of $B$ relative to $\left(\mathscr{H}_{t}\right)$.

Do the indifference prices, $\pi_{n}$, of an increasing sequence of filtrations converge to the indifference price, $\pi_{\infty}$, of their union? Before we provide sufficient conditions for the convergence, notice that if we enlarge the filtration, then a priori we do not know whether the indifference price will increase or decrease. This is illustrated in the next example.

Example 5.1. Let $U$ be a power utility function, i.e. $U(x)=x^{p}, 0<p<1$, for $x \geq 0$ and $U(x)=-\infty$ for $x<0$. Let $S_{t}=W_{t}+\alpha t$, where $\alpha>0$ and $W$ is a Brownian motion with respect to $\left(g_{t}\right)$.

First consider an endowment $B$ which is independent of $\left(g_{t}\right)$ and satisfies $\mathrm{P}(B=0)=\frac{1}{2}=$ $\mathrm{P}(B=-1)$. The indifference price, $\pi_{g}$, of $B$ under $\left(g_{t}\right)$ has to satisfy $u^{g}\left(1, B-\pi_{g}\right)=u^{g(1)}$. Observe that

$$
u^{g}\left(1, B-\pi_{g}\right)=u_{-\pi_{g}}^{g}\left(1, B-\pi_{g}\right) \leq \frac{1}{2} u^{g}\left(-\pi_{g}\right)+\frac{1}{2} u_{-\pi g}^{g}\left(1-\pi_{g}\right) .
$$


Now let $\mathscr{H}_{t}=\bigcap_{s>t} \sigma(B) \vee g_{s}$. If $\pi_{\mathscr{H}}$ is the indifference price under $\left(\mathscr{H}_{t}\right)$, then

$$
u^{\mathscr{H}}(1)=u^{\mathscr{H}}\left(1, B-\pi_{\mathscr{H}}\right)=\frac{1}{2} u^{g}\left(-\pi_{\mathscr{H}}\right)+\frac{1}{2} u^{g}\left(1-\pi_{\mathscr{H}}\right) .
$$

Note that $u^{\mathcal{H}}(1)=u^{g}(1)$ and, therefore, that

$$
\frac{1}{2} u^{g}\left(-\pi_{\mathcal{H}}\right)+\frac{1}{2} u^{g}\left(1-\pi_{\mathscr{H}}\right) \leq \frac{1}{2} u^{g}\left(-\pi_{g}\right)+\frac{1}{2} u_{-\pi_{g}}^{g}\left(1-\pi_{g}\right) .
$$

Since $\pi_{g}$ and $\pi_{\mathscr{H}}$ are negative, (5.1) can only be satisfied if $\pi_{g}<\pi_{\mathscr{H}}$.

Now let $\left(\tilde{\mathscr{H}}_{t}\right)$ be an enlargement of $\left(g_{t}\right)$ such that $u^{g}(x)<u^{\tilde{\mathscr{H}}}(x)$ for all $x>0$ and with respect to which there exists an optimal strategy $\theta^{*}$. Moreover, suppose that $S$ satisfies the NA condition with respect to $\left(\tilde{\mathscr{H}}_{t}\right)$. As an example, consider $\tilde{\mathscr{H}}_{t}=\bigcap_{s>t} \sigma\left(S_{T}+\psi\right) \vee g_{s}$, where the random variable $\psi$ is independent of $g_{T}$ and has the standard normal distribution.

Now let $\tilde{B}=\left(\theta^{*} \cdot S\right)_{T}$ be the optimal pay-off under $\left(\tilde{\mathscr{H}}_{t}\right)$. We claim that

$$
u^{\tilde{\mathscr{H}}}(x, \tilde{B})=u^{\tilde{\mathscr{H}}}(x)=u^{g}(x, \tilde{B}) .
$$

To prove this, suppose that $\eta \in \mathcal{A}(\tilde{\mathscr{H}})$ is a strategy such that

$$
\mathrm{E} U\left(x+(\eta \cdot S)_{T}+B\right)=\mathrm{E} U\left(x+\left(\left(\eta+\theta^{*}\right) \cdot S\right)_{T}\right)>-\infty .
$$

Note that $S$ does not allow arbitrage. Therefore, from Lemma 3.3, the strategy $\eta+\theta^{*}$ is $x$-admissible. Consequently, $\mathrm{E} U\left(x+(\eta \cdot S)_{T}+B\right) \leq u^{\tilde{\mathcal{H}}}(x)$. Hence,

$$
u^{\tilde{\mathscr{H}}}(x) \leq u^{\mathscr{g}}(x, \tilde{B}) \leq u^{\tilde{\mathscr{H}}}(x, \tilde{B})=x^{\tilde{\mathscr{H}}}(x)
$$

and, thus, (5.2) holds.

From (5.2) we immediately deduce that, under $\left(\tilde{\mathscr{H}}_{t}\right)$, the indifference price of $\tilde{B}$ is equal to 0 . However, $u^{g}(x)<u^{\tilde{\mathscr{H}}}(x)=u^{g}(x, \tilde{B})$, and the indifference price of $\tilde{B}$ with respect to $\left(g_{t}\right)$ has to be greater than 0 .

Henceforth, let $\left(g_{t}^{n}\right)$ again be an increasing sequence of filtrations converging to $\left(g_{t}^{\infty}\right)$ such that the assumptions of Section 2 are satisfied. Let $B$ be a random endowment. In this section, we will assume that, under $\left(g_{t}^{\infty}\right)$ and each $\left(g_{t}^{n}\right)$, the indifference prices of $B$ are always defined, and we denote them by $\pi^{\infty}$ and $\pi^{n}$, respectively. In our analysis of convergence we again start with the simpler case, with $\operatorname{dom}(U)=\mathbb{R}$.

\subsection{Convergence in the $\operatorname{case} \operatorname{dom}(U)=\mathbb{R}$}

We will need to make the assumption that

$$
u^{\infty}(x) \text { and } u^{\infty}(x, B) \text { are finite and strictly increasing on } \mathbb{R} .
$$

Note that (5.3) implies that the indifference prices $\pi_{n}$ and $\pi_{\infty}$ are defined.

Lemma 5.1. Suppose that (4.1) and (5.3) hold. Then the sequence of indifference prices $\left(\pi_{n}\right)$ is bounded from below and from above.

Proof. We first show that $\left(\pi_{n}\right)$ is bounded from above. Indeed, suppose that it is not. Then we can find a subsequence converging to $\infty$, and to simplify our notation we assume that 
$\lim _{n} \pi_{n}=\infty$. Concavity of the function $x \mapsto u^{\infty}(x, B)$ implies that $\lim _{x \rightarrow-\infty} u^{\infty}(x, B)=$ $-\infty$. Therefore,

$$
-\infty<u^{\infty}(x)=\lim _{n} u^{n}(x)=\lim _{n} u^{n}\left(x-\pi_{n}, B\right) \leq \lim _{n} u^{\infty}\left(x-\pi_{n}, B\right)=-\infty,
$$

which is a contradiction.

Now we show that $\left(\pi_{n}\right)$ is bounded from below. Suppose that there exists a subsequence converging to $-\infty$, and to simplify notation again assume that $\lim _{n} \pi_{n}=-\infty$. Let $k<\pi_{\infty}$. Then, from Theorem 4.1,

$$
\begin{aligned}
u^{\infty}(x)=\lim _{n} u^{n}(x) & =\lim _{n} u^{n}\left(x-\pi_{n}, B\right) \geq \lim _{n} u^{n}(x-k, B) \\
& =u^{\infty}(x-k, B)>u^{\infty}\left(x-\pi_{\infty}, B\right) \\
& =u^{\infty}(x),
\end{aligned}
$$

which is a contradiction. Consequently, the indifference prices $\pi_{n}$ all lie in a bounded interval.

Theorem 5.1. Suppose that (4.1) and (5.3) hold. Then $\lim _{n} \pi_{n}=\pi_{\infty}$.

Proof. By Lemma 5.1 the indifference prices $\pi_{n}$ are bounded, say by $C>0$. Suppose that $p \in[-C, C]$ is a cluster point of $\left(\pi_{n}\right)$, and let $\left(\lambda_{n}\right)=\left(\pi_{l(n)}\right)$ be a subsequence of $\left(\pi_{n}\right)$ converging to $p$. The concave functions $u^{n}(x, B)$ converge pointwise to $u^{\infty}(x, B)$. As a consequence, they converge uniformly on compact intervals (see Theorem 10.8 of [15]). Therefore, $\lim _{n} u^{l(n)}\left(x-\lambda_{n}, B\right)=u^{\infty}(x-p, B)$. Note that, in addition,

$$
\lim _{n} u^{l(n)}\left(x-\lambda_{n}, B\right)=\lim _{n} u^{l(n)}(x)=u^{\infty}(x)=u^{\infty}(x-\pi, B)
$$

and, hence, $u^{\infty}(x-p, B)=u^{\infty}(x-\pi, B)$. Since $u^{\infty}(x, B)$ is strictly increasing in $x$, we have $p=\pi$. As this is the case for any cluster point $p$ of $\left(\pi_{n}\right)$, the result holds.

The following lemma provides a sufficient condition for assumption (5.3) to be satisfied.

Lemma 5.2. Let $B$ be bounded. If $u^{\infty}(x)$ is finite and strictly increasing on $\mathbb{R}$, then so is $u^{\infty}(x, B)$.

Proof. Let $B$ be bounded by $C>0$. It is straightforward to show that $u^{\infty}(x, B)$ is concave and finite. Now suppose that it is not strictly increasing. It then has to be eventually constant, i.e. there exist constants $d$ and $e$ such that $u^{\infty}(x, B)=d$ for all $x \geq e$. Now observe that $u^{\infty}(x-C, B) \leq u^{\infty}(x) \leq u^{\infty}(x, B+C)$ and, therefore, that $u^{\infty}(x)=d$ for all $x \geq e+C$, which is a contradiction.

\subsection{Convergence in the $\operatorname{case} \operatorname{dom}(U) \neq \mathbb{R}$}

Let $\sup \{y: U(y)=-\infty\}=0$. In this subsection we fix $a>0$ and consider only $a$-admissible strategies. Let $B$ be an endowment such that essinf $B \geq-b, 0 \leq b<\infty$. We assume that

$u_{a}^{\infty}(x)$ and $u_{a}^{\infty}(x, B)$ are finite and strictly increasing on their respective domains.

Henceforth, $\pi^{n}$ and $\pi^{\infty}$ will denote the indifference prices determined by $u_{a}^{n}\left(x-\pi^{n}, B\right)=$ $u_{a}^{n}(x)$ and $u_{a}^{\infty}\left(x-\pi^{\infty}, B\right)=u_{a}^{\infty}(x)$, respectively.

Theorem 5.2. Let $x \geq a$ and suppose that (5.4) holds. If $x-\pi_{n} \geq a+b$ for all $n$, then the indifference prices satisfy $\lim _{n} \pi_{n}=\pi_{\infty}$. 
Proof. We again need to first show that $\left(\pi_{n}\right)$ is bounded from below. This can be done in exactly the same way as in the proof of Lemma 5.1. Suppose that $\lim _{n} \pi_{n}=-\infty$ and let $k<\min \left(x-a-b, \pi_{\infty}\right)$. Then, by Theorem 4.2, we have

$$
\begin{aligned}
u_{a}^{\infty}(x) & =\lim _{n} u_{a}^{n}\left(x-\pi_{n}, B\right) \geq \lim _{n} u_{a}^{n}(x-k, B) \\
& =u_{a}^{\infty}(x-k, B)>u_{a}^{\infty}\left(x-\pi_{\infty}, B\right) \\
& =u_{a}^{\infty}(x),
\end{aligned}
$$

which is a contradiction.

Note that $u_{a}^{n}(a+b, B) \geq U(a)>-\infty$ and, similarly, $u_{a}^{\infty}(a+b, B)>-\infty$. Consequently, we have uniform convergence of the concave functions on the compact interval $[a+b, C]$, where $C$ is the upper bound of $\left(x-\pi_{n}\right)$, and we deduce the result as in the proof of Theorem 5.1.

\section{Acknowledgements}

I thank Dirk Becherer for the idea of considering the convergence of indifference prices and an anonymous referee for many helpful remarks. This research was supported by the Deutsche Forschungsgemeinschaft through the SFB 649 'Economic Risk'.

\section{References}

[1] Amendinger, J., Becherer, D. And Schweizer, M. (2003). A monetary value for initial information in portfolio optimization. Finance Stoch. 7, 29-46.

[2] Ankirchner, S. (2005). Information and semimartingales. Doctoral Thesis, Humboldt-Universität zu Berlin. Available at http://edoc.hu-berlin.de/dissertationen/ankirchner-stefan-2005-07-01/PDF/Ankirchner.pdf.

[3] Ankirchner, S. And Imkeller, P. (2005). Finite utility on financial markets with asymmetric information and structure properties of the price dynamics. Ann. Inst. H. Poincaré Prob. Statist. 41, 479-503.

[4] Ankirchner, S., Dereich, S. and Imkeller, P. (2006). The Shannon information of filtrations and the additional logarithmic utility of insiders. Ann. Prob. 34, 743-778.

[5] Ansel, J.-P. and Stricker, C. (1992). Lois de martingale, densités et décomposition de Föllmer-Schweizer. Ann. Inst. H. Poincaré Prob. Statist. 28, 375-392.

[6] Delbaen, F. and Schachermayer, W. (1994). A general version of the fundamental theorem of asset pricing. Math. Ann. 300, 463-520.

[7] Delbaen, F. and Schachermayer, W. (2006). The Mathematics of Arbitrage. Springer, Berlin.

[8] Duffie, D. And Huang, C. (1986). Multiperiod security markets with differential information: martingales and resolution times. J. Math. Econom. 15, 283-303.

[9] Föllmer, H. And Imkeller, P. (1993). Anticipation cancelled by a Girsanov transformation: a paradox on Wiener space. Ann. Inst. H. Poincaré Prob. Statist. 29, 569-586.

[10] Jeulin, T. (1980). Semi-Martingales et Grossissement d'une Filtration (Lecture Notes Math. 833). Springer, Berlin.

[11] Mansuy, R. And Yor, M. (2006). Random Times and Enlargements of Filtrations in a Brownian Setting (Lecture Notes Math. 1873). Springer, Berlin.

[12] Pikovsky, I. and Karatzas, I. (1996). Anticipative portfolio optimization. Adv. Appl. Prob. 28, $1095-1122$.

[13] Protter, P. E. (2004). Stochastic Integration and Differential Equations (Appl. Math. New York 21), 2nd edn. Springer, New York.

[14] Revuz, D. And Yor, M. (1999). Continuous Martingales and Brownian Motion, 3rd edn. Springer, Berlin.

[15] Rockafellar, R. T. (1970). Convex Analysis (Princeton Math. Ser. 28). Princeton University Press. 